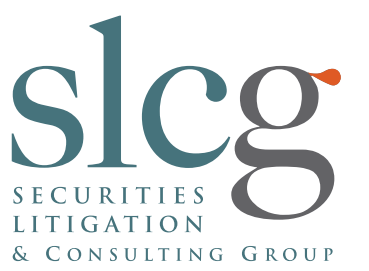

\title{
Modeling a Risk-Based Criterion for a Portfolio with Options*
}

\author{
Geng Deng, PhD, CFA, FRM ${ }^{\dagger} \quad$ Tim Dulaney, PhD, FRM ${ }^{\ddagger}$ \\ Craig McCann, PhD, $\mathrm{CFA}^{\S}$
}

December 13, 2013

\begin{abstract}
The presence of options in a portfolio fundamentally alters the portfolio's risk and return profiles when compared to an all equity portfolio. In this paper, we advocate modeling a risk-based criterion for optioned portfolio selection and rebalancing problems. The criterion is inspired by Chicago Mercantile Exchange's risk-based margining system which sets the collateralization requirements on margin accounts. The margin criterion computes the losses expected at the portfolio level using expected stock price and volatility variations, and is itself an optimization problem. Our contribution is to remodel the criterion as a quadratic programming subproblem of the main portfolio optimization problem using option Greeks. We also extend the margin subproblem to a continuous domain. The quadratic programming problems thus designed can be solved numerically or in closed-form with high efficiency, greatly facilitating the main portfolio selection problem. We present two extended practical examples of the application of our approach to obtain optimal portfolios with options. These examples include a study of liquidity effects (bid/ask spreads and limited order sizes) and sensitivity to changing market conditions. Our analysis shows that the approach advocated here is more stable and more efficient than discrete approaches to portfolio selection.
\end{abstract}

\section{Introduction and Motivation}

Various frameworks for appropriately addressing the risks inherent in portfolio allocations (not limited to portfolios with options) have been proposed. Value at Risk (VaR) and expected shortfall,

* (C)2013 Securities Litigation and Consulting Group, Inc.

${ }^{\dagger}$ Director of Research, Office: (703) 890-0741 Email: GengDeng@slcg.com

${ }_{\ddagger}^{\ddagger}$ Senior Financial Economist, Office: (703) 539-6777 or Email: TimDulaney@slcg.com

§President, Office: (703) 539-6760 or Email: CraigMcCann@slcg.com. 
also known as conditional VaR (CVaR), are two of many examples. VaR is essentially the value the portfolio could be expected to lose within a given time-horizon for a given probability. VaR is often criticized for not being a coherent risk measure due to the fact that VaR does not exhibit the property of sub-additivity. CVaR, a coherent alternative to VaR, determines the expected return considering only scenarios on the negative end of the return distribution. 1

There is a vast literature specializing in the optioned portfolio optimizations under various risk-management objectives/criterions. Alexander et al. (2006) show that, when derivatives are included in portfolio allocations, $\mathrm{VaR}$ and $\mathrm{CVaR}$ minimization problems are often ill-posed. By augmenting the traditional CVaR optimization to include a constraint based upon proportional cost, the authors show that it is possible to efficiently compute optimal solutions to this problem using a smoothing technique. Zhu et al. (2009) propose a robust portfolio optimization model that uses CVaR to tune a portfolio's exposure to return estimation risk by making the simplifying assumption that the covariance matrix is known.

Dert and Oldenkamp (2000) determine optimal portfolio allocation to portfolios including option positions by maximizing expected returns in the face of a short-fall constraint. Branger et al. (2008) compare optimal portfolio allocations to derivatives in the discrete time setting and the continuous time setting. The authors find that, in the discrete time case, optimal allocations are generally more conservative and, as a result, require less frequent rebalancing of portfolio allocations as compared to the continuous time case. By considering the higher order moments of option returns, Faias and Santa-Clara (2011) optimize portfolio allocations to option positions. Zymler et al. (2011) extend traditional robust portfolio optimization to include portfolios with options. The authors show that options can provide insurance-like guarantees to hedge the portfolio's exposure to returns outside of the uncertainty set considered during the robust optimization. Zymler et al. (2013) incorporate non-linear relationships between asset returns into worst-case VaR models to produce computationally tractable solutions to portfolio optimization problems involving derivative allocations. The authors show that the inclusion of options in a robust optimization framework can offer substantial benefits.

In this paper, we advocate a risk-based criterion that places a constraint on the largest expected loss a portfolio would experience within a reasonable range of variations in the values of the underlying assets and their volatilities. For an equity only portfolio, the potential losses are determined from possible variations in the market price alone. In a portfolio with options, price volatility plays an important role in portfolio value changes. This risk-based measure is inspired by the Chicago Mercantile Exchange's risk-based margining system.

Using such a risk-based measure has support in academic literature. By analyzing market data, Santa-Clara and Saretto (2009) study the effect of capital limits derived from margin requirements on the execution and profitability of writing out-of-the-money put options. The authors show that

1 For a review of expected shortfall, value-at-risk and other risk measures advocated in the literature, see Lleo (2009). For a comparative analysis of expected shortfall and VaR, see Yamai and Yoshiba (2002a) and Yamai and Yoshiba (2002b). 
the rules-based margin requirements imposed by the Chicago Board Options Exchange (CBOE) are more onerous than the risk-based margin requirements imposed by the Chicago Mercantile Exchange (CME). Murray (2013) also finds that margin requirements significantly decrease the realizable returns of short option positions.

In this risk-based approach to portfolio margining, the portfolio is stressed in several scenarios wherein the parameters of the underlying assets (asset price and/or volatility) are varied by prespecified amounts. In general, larger haircuts are applied to higher-risk, less-diversified, assets. ${ }^{2}$ This calculation methodology for the determination of margin requirements can be thought of as the other side of the coin from Regulation $\mathrm{T}(\operatorname{Reg} \mathrm{T})$ which codified the rules pertaining to the fraction of an exchange-traded security's current market value an agent was allowed to lend 3

The risk-based criterion is indeed a stand-alone discrete optimization problem - which we refer to as the optimization subproblem. Embedding such a criterion in a portfolio selection problem - the main problem - results in two levels of optimization. Moreover, the discrete nature of the stress-testing may lead to inaccurate stress test results. The main contribution of our paper is to reformulate the subproblem with several enhancements. First, we remodel the subproblem as a quadratic programming problem utilizing the Greeks as sensitivity measures for the options contained in the portfolio $4_{4}^{4}$ In addition, we expand the discrete domain to a continuous domain to more conservatively and accurately determine the portfolio risk. We summarize the approaches to solving the quadratic programming problem either in closed-form or numerically with a few iterations. The solution thus obtained in the subproblem is supplied to the main problem to facilitate optimal portfolio allocations.

The approach outlined in this paper has applications in a vast range of optimal portfolio allocation problems. We include two applications with several variations to illustrate the approach. The first example concerns an investor wanting to short a large number of straddles while respecting a risk-based constraint. The second example is an investor who has a more favorable view of a stock than the market. Given an amount of cash to invest and a set of option positions, we determine the portfolio that maximizes the expected gain for the holding period. We provide several variations to both examples such as adding constraints on the volatility, orders sizes and bid/ask spreads. By adding these constraints, we illustrate the stability and efficiency of our approach.

2 A haircut is simply another term used to describe the margin requirement or the maximum expected loss within a reasonable set of scenarios for a given position.

3 For a complete introduction to portfolio margining, see Rosenzweig (2006). For a comparative analysis of riskbased and rules/strategy-based margin methodologies as well as a more complete description of the historical evolution of margin requirements, see Coffman Jr. et al. (2010). For the possible effects of the recent Dodd-Frank Act on portfolio margin utilization, see Filler (2010).

4 We explicitly consider the Greeks derived from the Black-Scholes model in this paper. As a result, the paper has certain limitations in the perception of risks (e.g. lack of skewness and price jumps, etc.). Extending this paper to more empirically realistic models is the subject of future research. 


\section{General Framework}

Let our working portfolio consist of a set of $J$ assets $\left\{S_{j} \mid j=1,2, \cdots, J\right\}$ and options on those assets. We denote the price of the securities in the portfolio by $P_{i, j}$ where $P_{1, j}$ denotes the price of asset $j$ and $P_{i, j}$ represents the price of option $i(i>1)$ on underlying asset $j$. Similarly, the portfolio position in asset $j$ is given by $n_{1, j}$ and the portfolio position in option $i$ on asset $j$ is defined to be $n_{i, j}$.

The main optimization problem we address in this paper is generally given by

$$
\min _{n_{i, j}} f\left(n_{i, j}\right) \text { such that } \operatorname{NLV}\left(n_{i, j}\right) \geq \text { Potential Loss }\left(n_{i, j}\right),
$$

where $f(\cdot)$ is a generic objective function associated with the goal for the portfolio selection problem (e.g., maximizing returns, minimizing risk or minimizing transaction costs, etc.). NLV(·) represents the net liquidation value of the portfolio and is a function of the positions in the underlying securities. In Section 4, we will provide two specific applications to clarify the utility of our approach. $5^{5}$

The net liquidation value of a portfolio is the sum of the market value of all portfolio positions

$$
\operatorname{NLV}\left(n_{i, j}\right)=\sum_{i, j} P_{i, j} n_{i, j}+C,
$$

where $C$ represents the cash position in the portfolio ${ }^{6}$ Note that in a portfolio rebalancing process, where no cash inflows or outflows exist, the NLV is a constant regardless of any changes portfolio positions 7 The "potential loss" is the maximum loss the portfolio would sustain given a reasonable range of market parameter variation (asset prices and volatilities). 8 Since we work on a portfolio with options, asset volatilities are also crucial in determining the market values of the securities. The variation of these parameters and resulting analysis of portfolio value changes is the essence of stress testing.

The Chicago Mercantile Exchange's margining system uses a set of discrete scenarios to stress test portfolios. Let $\mathbb{S}=\{1,2, \ldots, S\}$ be the finite set of scenarios with unique parameter changes $\vec{x}_{j}^{k}=\left(\frac{\Delta S_{j}}{S_{j}}, \frac{\Delta \sigma_{j}}{\sigma_{j}}\right)_{k}^{T}, k \in \mathbb{S}$, for asset $j$. A graphical depiction of these scenarios can be found in Figure 1. Among the finite set of candidate changes $\vec{x}_{j}^{k}, k \in \mathbb{S}, \vec{x}_{j}^{*}$ corresponds to the largest potential loss the portfolio can expect to experience given a reasonable range within which the stock price and volatility can be expected to change.

5 There may be other constraints in the problem, but for illustrative purposes we only show the risk-based constraint. We will show examples of various constraints in Section 4

6 As a first approximation, we assume for present purposes that the securities in the portfolio are sufficiently liquid to ignore secondary effects and bid-ask spreads.

7 In reality, the NLV will decrease as a result of transactions costs associated with commissions or bid/ask spreads.

8 In the context of portfolio margin requirements implemented by the Options Clearing Corporation, these ranges vary from $6-8 \%$ for broad-based indexes to $15 \%$ for equities. 
Figure 1: Scenarios considered in the conventional risk-based haircuts methodology. Given asset $j$, the $\mathrm{X}$-axis represents changes in the asset price and the $\mathrm{Y}$-axis represents the changes in the volatility.

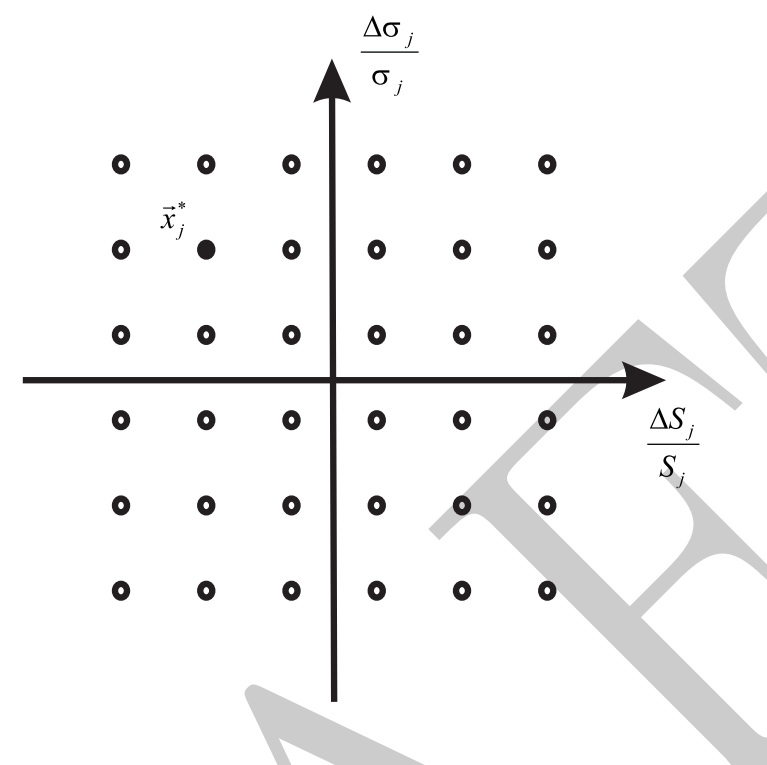

If $\vec{x}_{j}^{*}$ is defined as the scenario under which the portfolio incurs the largest loss, then it solves the following optimization subproblem

$$
\vec{x}_{j}^{*}=\arg \min _{k \in \mathbb{S}} \Delta V_{j}\left(\vec{x}_{j}^{k} \mid n_{i, j}\right)
$$

where the change in value of a portfolio is given by,

$$
\Delta V_{j}\left(\vec{x}_{j} \mid n_{i, j}\right)=\sum_{i}\left(P_{i, j}\left(\vec{x}_{j}\right)-P_{i, j}(0)\right) n_{i, j} .
$$

The maximum potential loss over the scenarios considered is given by the sum of the largest loss of each asset

$$
\text { Potential Loss }\left(n_{i, j}\right)=-\sum_{j} \Delta V_{j}\left(\vec{x}_{j}^{*} \mid n_{i, j}\right) .
$$

In most cases, the change $\vec{x}_{j}^{*}$ corresponding to the worst-case scenario is located on the boundary of the discrete domain, but it is also possible that it is located inside the boundary - for example, a portfolio containing only an at-the-money straddle. Since there are long and short option positions in the portfolio, the objective function $\Delta V_{j}\left(\vec{x}_{j} \mid n_{i, j}\right)$ may not be concave or convex with respect to $\frac{\Delta S_{j}}{S_{j}}$ or $\frac{\Delta \sigma_{j}}{\sigma_{j}}$. As a result, the function may have multiple local minima in the domain. The potential loss calculation is conceptually similar to the VaR measure. The potential loss gives the worst possible loss over a range of market conditions. On the other hand, VaR gives the worst possible loss given a probability of occurrence. 
The primary benefit of the discrete scenario-based approach is its simplicity - determine the objective value of each scenario and choose the smallest. On the other hand, the discrete setting makes the main problem (1) numerically intractable for several reasons:

- The problem embeds two levels of optimization. A discrete subproblem needs to be solved in each iteration of the main optimization problem.

- The worst-case scenario solved via the subproblem described above is discrete. The discontinuities in solutions result in a cumbersome main problem.

- The accuracy of the solutions depends on the mesh size of the discrete set and as a result the grid could miss a local minimum that occurs between scenarios. By missing such minima, the estimated portfolio stress test may not provide a precise picture of the risks inherent in the portfolio allocations.

- The condition derived in the discrete setting does not provide useful information on the constraint surface (such as gradients or Hessians) which could be used in the main optimization problem.

- Increasing the number of scenarios increases accuracy, but at the cost of efficiency.

Given the limitations on efficiency and accuracy that the traditional discrete optimization exhibits in this subproblem, we suggest a continuous extension that improves the efficiency of computing the risk-based constraint. Although the risk-based scenarios consider an uncountably infinite number of scenarios in the continuous extension, by using advances in optimization we can efficiently solve these problems with precision.

\section{Approaches to Risk-Based Constraint Estimation}

In order solve the optimization subproblem (2) efficiently, we proceed with several modifications described in the following subsections.

- Approximate the objective with a quadratic function. When options are involved, the valuations are typically complex to compute. A quadratic approximation enhances the tractability of the problem without significant loss of precision for many portfolios.

- Extend the discrete domain to a continuous set. We consider two types of continuous sets here: a circle set and a box set. This approach converts the discrete-optimization problem into a continuous optimization problem and avoids the mesh size limitations discussed in the previous section. The optimal solution to the subproblem changes continuously when the underlying portfolio is altered. An ancillary benefit of our extension is that the solution constrained within the circle has continuous changes. Small variations in the positions $n_{i, j}$ for 
the main optimization problem results in small changes of optimal scenario $\vec{x}_{j}^{*}$ determining the risk-based criterion.

Although we summarize the approach of solving the subproblems and main optimization problems in each subsection, this is not the main focus of our contribution. We use our approaches to point out inefficiencies in the conventional approach while advocating for an alternative that preserves precision.

\subsection{Quadratic Approximation to Objective Function}

In CME margin calculations and stress tests, the Black-Scholes model is used to compute the value of options. For a given asset $j$, the value of the asset and its derivatives are

$$
P_{i, j}= \begin{cases}S_{j} & i=1 \\ O\left(S_{j}, \sigma_{i}, K_{i}, r, q, T_{i}, C P_{i}\right) & i>2\end{cases}
$$

In particular, we have

$$
O(S, \sigma, K, r, q, T, C P)= \begin{cases}C(S, \sigma, K, r, q, T) & C P=\text { Call } \\ P(S, \sigma, K, r, q, T) & C P=\text { Put }\end{cases}
$$

and explicit formulas for these functions can be found in Appendix A.

Greeks are useful measures for option price sensitivities to changes in market parameters such as stock price, volatility, interest rates, and maturity. The Black-Scholes model endows analysts with an additional tool through analytic closed-form solutions for these sensitivities? Therefore the portfolio value change under parametric changes $\vec{x}_{j}$ can be approximated neatly with a quadratic function. This is very similar to a Taylor expansion of the value function $V\left(\vec{x}_{j} \mid n_{i, j}\right)$ to second order about the point $\vec{x}=(0,0)^{T}$.

In terms of the objective function in (2), the change in value of any securities associated with asset $j, \Delta V_{j}$, to second order in $\vec{x}_{j}$ is estimated by

$$
\Delta \widetilde{V}\left(\vec{x}_{j} \mid n_{i, j}\right)=\vec{g}_{j}^{T} \vec{x}_{j}+\frac{1}{2} \vec{x}_{j}^{T} B_{j} \vec{x}_{j},
$$

where $\vec{g}_{j}^{T}=\left(\sum_{i} D_{i, j} n_{i, j}, \sum_{i} V_{i, j} n_{i, j}\right), \vec{x}_{j}=\left(\frac{\Delta S_{j}}{S_{j}}, \frac{\Delta \sigma_{j}}{\sigma_{j}}\right)^{T}$ and

$$
D_{i, j}=\frac{\partial P_{i, j}}{\partial S_{j}} S_{j} \quad \text { and } \quad V_{i, j}=\frac{\partial P_{i, j}}{\partial \sigma_{j}} \sigma_{j}
$$

9 Further motivation for the use of the Black-Scholes model is found in the regulatory use of this model when determining risk-based margin requirements. 
The partial derivatives in the equations above are the conventional Greeks delta and vega. The symmetric $2 \times 2$ matrix $B_{j}$ is defined as follows

$$
B_{j}=\sum_{i}\left(\begin{array}{cc}
\frac{\partial^{2} P_{i, j}}{\partial S_{j}^{2}} n_{i, j} & \frac{\partial^{2} P_{i, j}}{\partial S_{j}, \sigma_{j}} n_{i, j} \\
\frac{\partial^{2} P_{i, j}}{\partial \sigma_{j} \partial S_{j}} n_{i, j} & \frac{\partial^{2} P_{i, j}}{\partial \sigma_{j}^{2}} n_{i, j}
\end{array}\right) .
$$

Note that for the stock component, $i=1$, all second derivatives vanish. The matrix $B_{j}$ is not necessarily positive semi-definite, which implies the objective function $\Delta \widetilde{V}\left(\vec{x}_{j} \mid n_{i, j}\right)$ may not be convex or concave.

For small enough parametric changes in the case of at-the-money options - or for portfolios that contain options that are sufficiently far-from-the-money, and therefore relatively insensitive to implied volatility changes - this approximation of portfolio value changes is very precise. In fact, the first order change in portfolio value could be sufficient for some purposes. Though we leave the extension to higher order terms in $\vec{x}_{j}$ to future research, the extension is straight-forward.

\subsection{Continuous Extension of the Domain}

Instead of using a discrete domain $\mathbb{S}$, the subproblem (2) can be extended to a continuous analog with $\vec{x}_{j}^{*} \in \mathbb{S}_{c}=\{\vec{x} \mid\|\vec{x}\| \leq c\}$. The optimization problem is defined by

$$
\vec{x}_{j}^{*}=\arg \min _{\vec{x}_{j} \in \mathbb{S}_{c}} \Delta V_{j}\left(\vec{x}_{j} \mid n_{i, j}\right) \approx \arg \min _{\vec{x}_{j} \in \mathbb{S}_{c}} \vec{g}_{j}^{T} \vec{x}_{j}+\frac{1}{2} \vec{x}_{j}^{T} B_{j} \vec{x}_{j} .
$$

The benefit of this approach is that the problem becomes the well-known "trust-region" problem in optimization literature (Nocedal and Wright, 1999). Given the vast literature on techniques to solve the trust-region problem, the optimal solution can be efficiently computed. In Figure 2, we illustrate the solution $\vec{x}_{j}^{*}$ computed within the new domain $\vec{x}_{j} \in \mathbb{S}_{c}$.

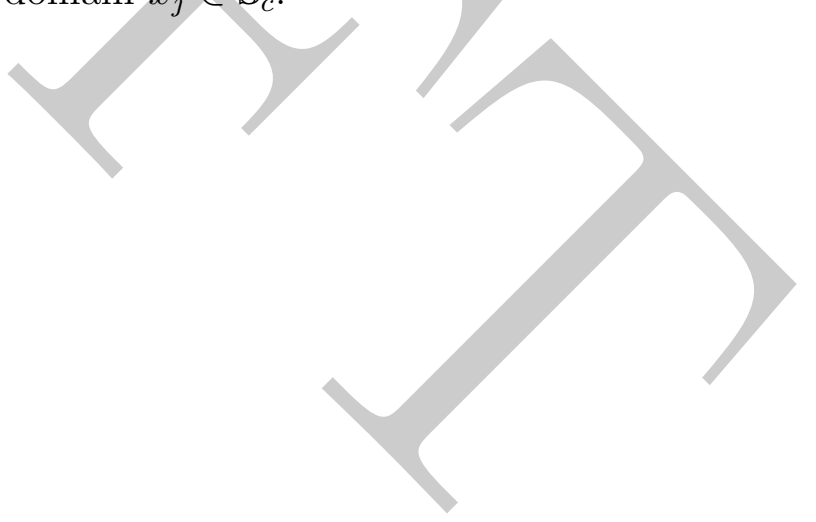


Figure 2: A continuous generalization of the discrete risk-based haircuts methodology.

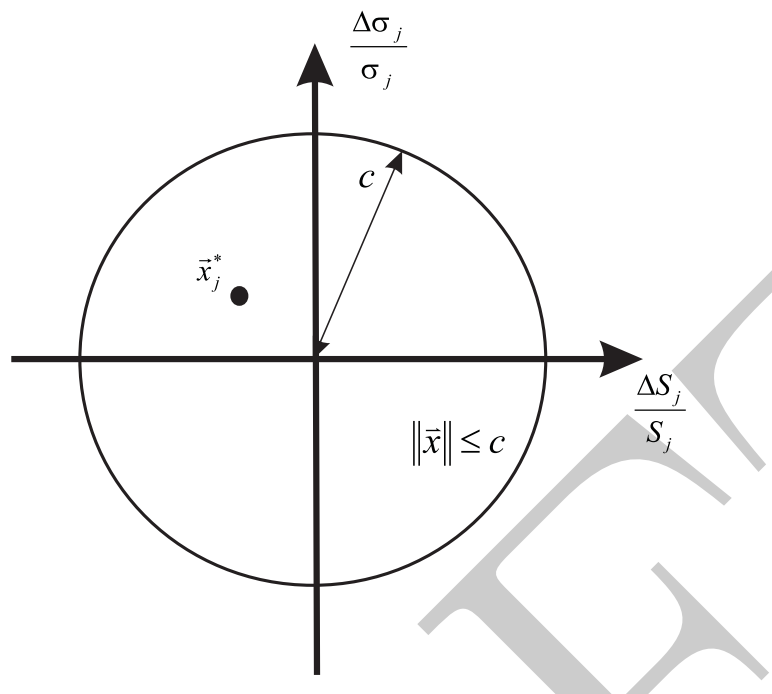

The following theorem outlines a conventional approach to determine the solution of this trust region problem.

Theorem 1. The vector $\vec{x}^{*}$ is a global solution of the trust-region problem

$$
\min _{\vec{x} \in \mathbb{R}^{n}}\left(\vec{g}^{T} \vec{x}+\frac{1}{2} \vec{x}^{T} B \vec{x}\right) \quad \text { such that }\|\vec{x}\| \leq c
$$

if and only if $\vec{x}^{*}$ is feasible and there is a scalar $\lambda \geq 0$ such that the following conditions are satisfied:

$$
\begin{aligned}
& (B+\lambda I) \vec{x}^{*}=-\vec{g}, \\
& \lambda\left(c-\left\|\vec{x}^{*}\right\|\right)=0, \\
& (B+\lambda I) \text { is positive-semidefinite. }
\end{aligned}
$$

where $I$ is the $n \times n$ identity matrix.

An efficient way to solve the trust-region subproblem is to use the "exact trust-region" method in Moré and Sorensen (1983) and Nocedal and Wright (1999), which takes normally less than 5 iterations for a two-dimensional problem like this. The method is proven to converge to the near exact global solution with very high probability. An alternative and simpler method to solve the problem is an indefinite version of the dog-leg algorithm (Powell, 1970; Byrd et al., 1988), which uses only two steps to determine an approximate solution. Since only two steps are involved, one can derive the solution in closed-form. Using the closed-form solution of the subproblem, gradient and Hessian information can be derived for the main problem. With these analytic properties, the main optimization problem is not limited to the use of a derivative-free or a derivative-estimation solver. 


\subsection{Alternative Extension of the Risk-Based Constraint Calculation}

As an alternative to the above continuous extension, consider the problem with $\vec{x}_{j}^{*}$ defined by

$$
\vec{x}_{j}^{*}=\arg \min _{\vec{x}_{j} \in \mathbb{S}_{\infty}} \Delta V_{j}\left(\vec{x}_{j} \mid n_{i, j}\right) .
$$

where the scenarios under consideration fall within the rectangular region specified by $\mathbb{S}_{\infty}=\{\vec{x} \mid$ $\left.\|\vec{x}\|^{\infty} \leq c\right\}$ as depicted in Figure 3 .

Figure 3: An alternative continuous generalization of the discrete risk-based haircuts methodology.

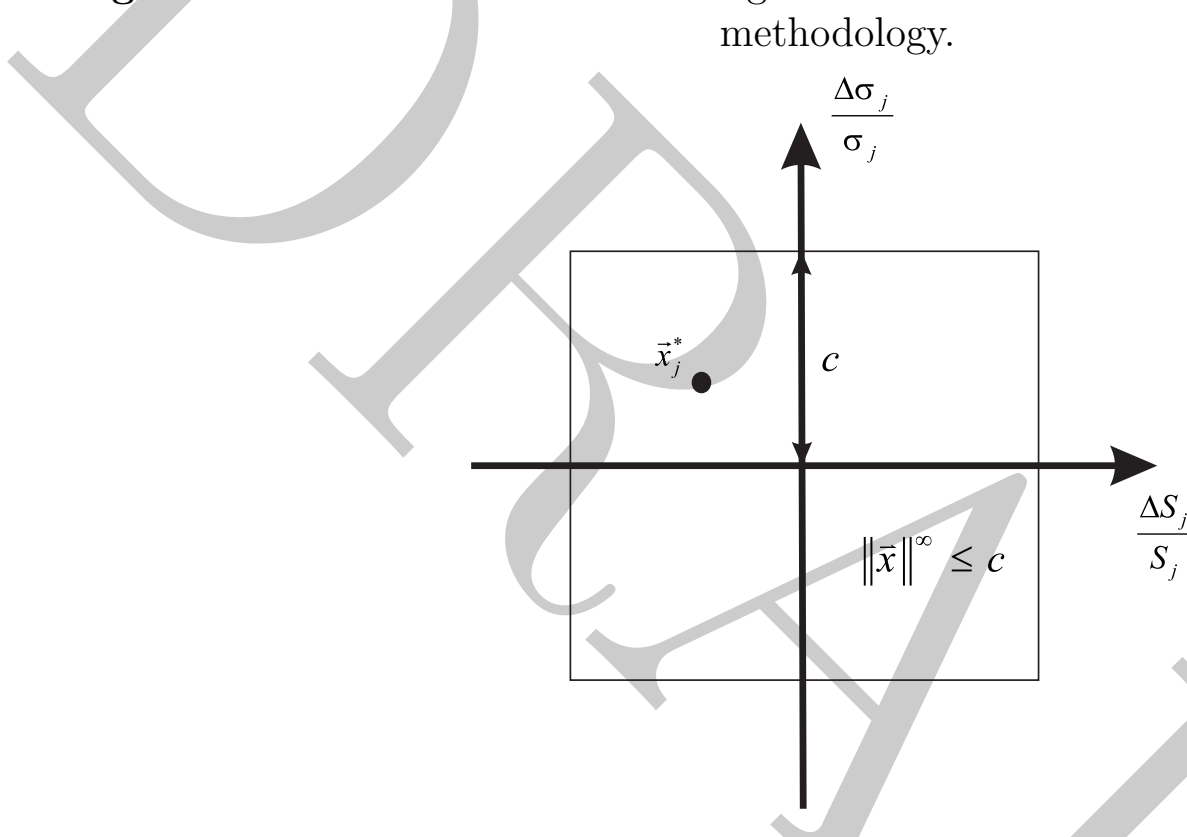

For problems of this type (in which variables vary within an interval), the gradient-projection method is especially efficient and precise (Nocedal and Wright, 1999). The following essentially states the KKT conditions for the optimization problem. The box constraint $\|\vec{x}\|^{\infty} \leq c$ is equivalent to a standard set of constraints

$$
\vec{a}_{k}^{T} \vec{x} \geq-c, k \in \mathcal{I}=\{1,2,3,4\} \text {, where } A=\left(\begin{array}{c}
\vec{a}_{1}^{T} \\
\vec{a}_{2}^{T} \\
\vec{a}_{3}^{T} \\
\vec{a}_{4}^{T}
\end{array}\right)=\left(\begin{array}{rr}
1 & 0 \\
-1 & 0 \\
0 & 1 \\
0 & -1
\end{array}\right) \text {. }
$$

The KKT conditions for the box region problem

$$
\min _{\vec{x} \in \mathbb{R}^{n}} \vec{g}^{T} \vec{x}+\frac{1}{2} \vec{x}^{T} B \vec{x}, \quad \text { such that } \vec{a}_{k}^{T} \vec{x} \geq \vec{c}, \quad \text { for all } k \in \mathcal{I}
$$


define the optimal solutions to the problem

$$
\begin{aligned}
B \vec{x}^{*}+\vec{g}-\sum_{k \in \mathcal{A}\left(\vec{x}^{*}\right)} \lambda_{k}^{*} a_{k} & =0, \\
\vec{a}_{k}^{T} \vec{x}^{*} & =-c, \text { for all } k \in \mathcal{A}\left(\vec{x}^{*}\right), \\
\vec{a}_{k}^{T} \vec{x}^{*} & \geq-c, \text { for all } k \in \mathcal{I} \backslash \mathcal{A}\left(\vec{x}^{*}\right), \\
\lambda_{k}^{*} & \geq 0, \text { for all } k \in \mathcal{A}\left(\vec{x}^{*}\right),
\end{aligned}
$$

where $\mathcal{A}\left(\vec{x}^{*}\right)$ is an active set at the optimal solution $\vec{x}^{*}$ defined as

$$
\mathcal{A}\left(\vec{x}^{*}\right)=\left\{k \in \mathcal{I} \mid \vec{a}_{k}^{T} \vec{x}^{*}=-c\right\} .
$$

We solve the optimization problem using the gradient-projection method - see, for example, Nocedal and Wright (1999). The convergence for the method is very fast, typically within two iterations in our two-dimensional problem. Analogous to the previously discussed extension, the solution $\vec{x}_{j}^{*}$ is represented in closed-form as a function of $n_{i, j}$. When plugging back to the main optimization problem, the constraint itself can be expressed as a function of portfolio positions. From these closed-form expressions, gradients and Hessian's can be derived.

\section{Applications}

In this section, we work through a few examples of the risk-based optimization approaches outlined above. In each case, we show the optimal portfolio allocation for each approach and discuss the salient features 10

\subsection{Shorting a Straddle Position}

In this example, we consider an investor who would like to implement a short straddle position on an asset because she believes the underlying asset will not change significantly before the options expire in three months. She would like to short as large a position as possible, but the margin system limits the maximum number of option contracts she can write.

The options are at-the-money with strike price of $\$ 60$. We assume the underlying asset pays a constant and continuously compounded dividend of $1 \%$ and that the risk-free rate is a constant and continuously compounded $3 \%$. We assume that both the put and the call option have prices that imply a Black-Scholes volatility of $20 \%$.

Given that the investor has $\$ 100,000$ in cash to collateralize her short position, we would like to know how many straddles she can sell short while limiting her potential for losses in the next

10 The particular securities chosen in each example are sensitive to market conditions. Sudden and dramatic changes in market conditions can lead to entirely different results. 
observation period. Let $N$ be the number of straddles the investor will short and let $C(K, T)$ and $P(K, T)$ represent the call and put options comprising the straddle ${ }^{11}$ The optimization problem to solve in this case is therefore

$$
\arg \max f(N)=N
$$

subject to the constraint

$$
N L V(N) \geq \text { Potential } \operatorname{Loss}(N) .
$$

The net liquidation value (NLV) is a function of the number of straddles written $(N)$ and it is a constant $N L V(N)=\$ 100,000$. The potential loss given $N$ straddles in the portfolio. For now, we consider variations of the implied volatility and underlying asset price to be bounded by 15\% 12 The main optimization problem is setup in MATLAB using the routine fmincon, which implements either a trust-region-reflective algorithm or an active-set algorithm to solve the nonlinear optimization problem. The optimization problem is only a one dimensional problem and is relatively easy to solve. On an Intel 4th generation i7 CPU machine, the optimization code was completed within a few seconds, even under the circumstances that 500 function evaluations were used.

The efficiency of each portfolio optimization given the risk-based constraints depends on the starting position - the seed position for the optimization - and the approach. Table 1 summarizes number of function valuations used for each risk-based constraint optimization. The discrete scenario-based model shows much more volatility in the number of function valuations required to obtain the optimal solution.

Table 1: Optimal number $(N)$ of short straddles given $\$ 100,000$ cash to collateralize the option writing. Function evaluation ranges result from different starting positions.

\begin{tabular}{lcc}
\hline \hline Approach & $N$ & Function Evaluations \\
\hline Discrete & 19,087 & 14 to 500 \\
Circle & 16,440 & 11 to 84 \\
Box & 14,764 & 11 to 176 \\
\hline \hline
\end{tabular}

The discrete calculation results in the least conservative portfolio because the scenarios considered by this approach are a subset of those of the continuous box extension. Similarly, the box extension is more conservative than the circular extension since all scenarios considered in the circular extension are also considered in the box extension (the circle is inscribed in the square).

${ }^{11}$ In this case, $n_{1,1}=0$ since the investor does not hold a long or short position in the underlying and $n_{2,1}=n_{3,1}=$ $-N$ representing equal short positions in the call and put options.

${ }^{12}$ The use of $15 \%$ variation is in line with regulatory agency definitions. In both examples, we use $15 \%$ as the variation level. In fact, we do not recommend applying our method for variations greater than $15 \%$, because we rely on accuracy of quadratic expansion/approximation, which is only valid for small variations. 
At the same time, the continuous extensions also determine their respective optimal portfolios more efficiently-with much fewer function valuations. The performance, described by the number of function evaluations, for the discrete scenario optimization problem shows more variance and, in some cases, the optimization becomes very slow.

Since the optimization procedures used in this paper are static and therefore reveal nothing about the dynamics of asset prices. By observing the changes in the static results for various levels of the current asset price and implied volatility, the next two analyses shed light on the sensitivity of optimization results to changing market conditions.

Sensitivity to stock price. The levels of stock prices affect the optimal number of straddles shorted. This sensitivity test checks the robustness of the solutions when market conditions change. In Figure 4, we show the optimal number of straddles to short in each scenario as a function of the underlying stock price.

Figure 4: Optimal number of short straddles as a function of stock prices.

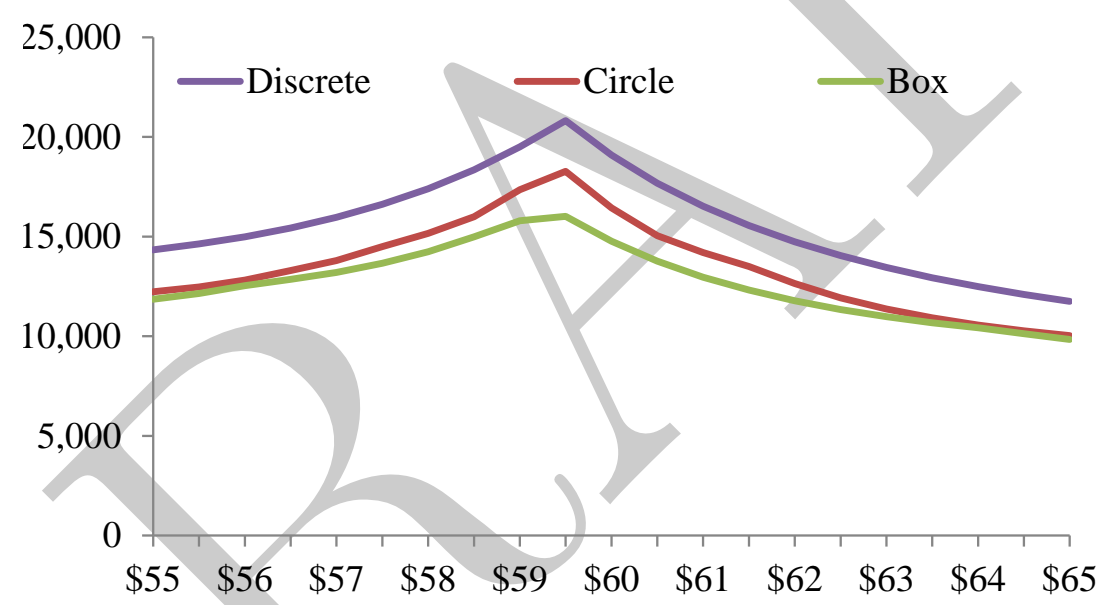

Since the straddle has strike of $\$ 60$, a larger number of straddles can be purchased when the stock price is near $\$ 60$. The lines contained in Figure 4 represent solutions that satiate the NLV constraint. More conservative portfolios would short fewer options and experience less turnover when compared with portfolios that satiate the NLV constraint.

Changing the underlying stock value does not affect the computational efficiency of the optimization approaches studied. In particular, we observe that the optimization procedures use approximately the same number of the function valuations.

Sensitivity to volatility. Since portfolio allocation is also sensitive to the level of implied volatility, we explore the effect of varying the volatility on the optimal number of straddles to 
short in each optimization algorithm. We vary the implied volatility of the options from $15 \%$ to $25 \%$ and plot the results in Figure 5 .

Figure 5: Optimal number of short straddles as a function of implied volatility

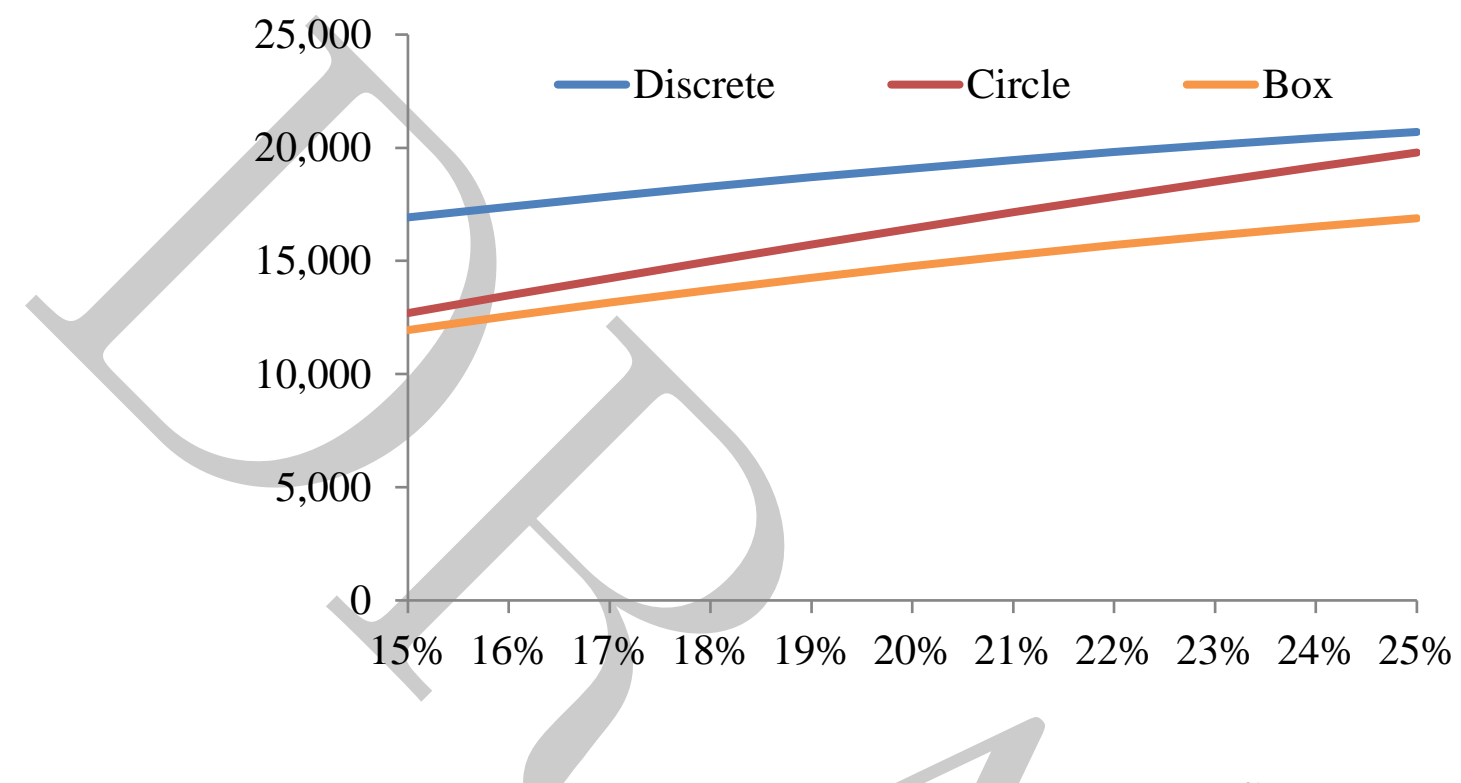

The results are relatively insensitive to changes in implied volatility. Generally speaking, a more volatile stock actually results in optimal portfolios with higher leverage. This is because the option prices are more sensitive to changes in volatility in a low volatility environment than a high volatility environment.

Sensitivity to positions. It is possible that the optimization algorithm would be sensitive to the positions in the portfolio. To study the effect of the positions in the portfolio, we adjust the strategy from a straddle to a strangle such that both options are out-of-the-money - increasing the call option strike to $\$ 65$ and decreasing the put option strike to $\$ 55$, while keeping the underlying asset price fixed at $\$ 60$. A short strangle is generally betting stock price will not close beyond the range defined by the two strike prices $(\$ 55$ and $\$ 65)$. Table 2 summarizes the results of the various optimization approaches given this alternative strategy. 
Table 2: Optimal number $(N)$ of short strangles given $\$ 100,000$ cash to collateralize the option writing. Function evaluation ranges result from different starting positions.

\begin{tabular}{lcc}
\hline \hline Approach & $N$ & Function Evaluations \\
\hline Discrete & 22,855 & 14 to 357 \\
Circle & 22,327 & 14 to 40 \\
Box & 19,861 & 11 to 40 \\
\hline \hline
\end{tabular}

The algorithms find that one can short more strangles for a few reasons. First, the options are out-of-the-money and therefore less expensive than the positions that made up the straddle. In addition, the options are less sensitive to price and volatility changes than those involved in the at-the-money straddle.

\subsection{Maximizing Expected Holding Period Returns}

This is an example in an upward trending market, in which the investor believes that the asset will appreciate more quickly than the general market implies. As a result, the investor would like to maximize her exposure to the asset using a fixed amount of cash to purchase/sell short-term call and put options as well as stock. The investor would view the stock and call options as cheap and put options as expensive since her view of the stock is generally more favorable than that of the market.

For our example, the stock has a current market value of $\$ 50$. Besides the stock, there is a set of four options (written on the same stock) in which the investor can invest: two call options and two put options. The maturities for the options are either one month or three months. For call options, the strike prices are $\$ 60$ and for put options, the strike prices are $\$ 40$. See Table 3 for a detailed list of options. Initially, the investor has $\$ 100$ cash to purchase or collateralize any position in the stock or options. Since we allow long and short positions for options, the amount of initial cash is not a crucial factor for option allocations. However, the amount of initial cash controls the strictness of the stress testing criterion.

We assume that the investor expects the non-dividend paying stock to appreciate at $5 \%$ annually, which is greater than the annualized risk-free rate of $3 \%$. We assume that the stock has a continuously compounded constant dividend yield of $1 \%$, and all the put and the call options have prices that imply a Black-Scholes volatility of $20 \%$.

We have considered here only a "flat" volatility smile for ease of presentation and parametric simplicity. We have explored including more realistic volatility smiles, but we find that this extension does not decrease efficiency of the optimization approaches considered here. Including such complications is as simple as changing input parameters.

We implement our optimization algorithm to determine the optimal number of options the 
investor will purchase or short in order to maximize her portfolio's expected return over the next month ${ }^{13}$

$$
\max _{n} f\left(n_{i, 1}\right)=\max _{n} \mathbb{E}_{t}\left[\text { Portfolio Value }\left(n_{i, 1}\right)\right], i \in\{1,2,3,4,5\}
$$

The optimization model imposes the single constraint

$$
N L V\left(n_{i, 1}\right) \geq \text { Potential } \operatorname{Loss}\left(n_{i, 1}\right)
$$

Using the same definition we used in Section 2, the risk-based margin criterion prohibits the investor from taking very aggressive long and short positions.

The maximization algorithm for the continuous extensions result in the portfolio allocations summarized in Table 3 ,

Table 3: Portfolio allocations resulting from optimization algorithms.

\begin{tabular}{cccccc}
\hline \hline \multirow{2}{*}{ Security Type } & $\begin{array}{c}\text { Months to } \\
\text { Expiration }\end{array}$ & $\begin{array}{c}\text { Strike } \\
\text { Price }\end{array}$ & $\begin{array}{c}\text { Scenario } \\
\text { Based }\end{array}$ & $\begin{array}{c}\text { Circle } \\
\text { Extension }\end{array}$ & $\begin{array}{c}\text { Box } \\
\text { Extension }\end{array}$ \\
\hline Call & 1 & $\$ 60$ & 435 & 423 & 435 \\
Call & 3 & $\$ 60$ & 2,150 & 2,164 & 2,133 \\
Put & 1 & $\$ 40$ & 30 & 28 & 30 \\
Put & 3 & $\$ 40$ & -941 & $-1,051$ & -945 \\
Stock & & & -26 & -32 & -30 \\
\hline Expected Portfolio Value & & $\$ 111.07$ & $\$ 110.72$ & $\$ 110.60$ \\
\hline \hline
\end{tabular}

The optimal portfolio allocation resulting from the optimization algorithm is such that the investor shorts longer-dated put options and stock in order to leverage her exposure to the stock with longerdated call options. The scenario-based portfolio has the largest holding period return as a result of the least conservative allocation. The most conservative portfolio, the result of the box extension, also has the lowest holding period return.

For either of the continuous extensions, the convergence to the optimal solution is generally robust, tested from different starting positions. The discrete optimization, on the other hand, is relatively unstable when changing the starting location for the optimization. In addition, the discrete optimization has a tendency to get stuck in local minima and, as a result, the efficiency of the optimization algorithm decreases dramatically.

The optimization problem contains only five variables. The typical number of solutions ranges from 100 to 2000. We consistently observed that the scenario-based approach takes three times as many function evaluations as the circle or the box based approach. On an Intel 4th generation

${ }^{13}$ Our approach of maximizing holding period returns, rather than risk adjusted returns, is motivated in part by the literature - see (Dert and Oldenkamp, 2000). 
i7 CPU machine, the code took several minutes to complete. The majority of the computation power was spent on evaluations of the objective function, which involves calculating the expected return - an integration over normal distribution. Instead of using numerical integration to compute the expectation value, we used Gauss-Hermite quadrature to approximate the expected value in integration form.

Our test shows that if the problem does not utilize the gradient information either in the objective function then an optimization problem could fail to converge to solution when variable size (number of assets) is more than 30. With gradient information, the optimization could be solved in a few minutes for a 100 -variable problem.

Adding a new volatility/risk constraint. We can alter the optimization problem by imposing a strict risk constraint on the expected volatility of portfolio returns and perform the same computation. The constraint specifies that the volatility of the return should be less than $50 \%$.

$$
\operatorname{std}\left(\operatorname{Portfolio} \operatorname{Return}\left(n_{i, 1}\right)\right) \leq 50 \%
$$

where

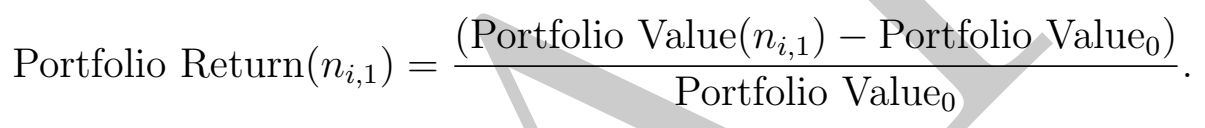

Implementing both the new constraint and the constraint (18) - we have the results summarized in Table 4. The results show that adding the strict risk criterion results in more conservative portfolios with lower returns. This example shows that the optimization problem can be easily expanded to satisfy more constraints.

Table 4: Portfolio allocations resulting from optimization algorithms (with a

\begin{tabular}{cccccc}
\multicolumn{7}{c}{ volatility/risk constraint). } \\
\hline \hline \multirow{2}{*}{ Security Type } & Months to & Strike & Scenario & Circle & Box \\
& Expiration & Price & Based & Extension & Extension \\
\hline Call & 1 & $\$ 60$ & -4 & -17 & -3 \\
Call & 3 & $\$ 60$ & 241 & 180 & 246 \\
Put & 1 & $\$ 40$ & 66 & 55 & 55 \\
Put & 3 & $\$ 40$ & -406 & -436 & -420 \\
Stock & & & -1 & 3 & -1 \\
\hline Expected Portfolio Value & & 101.98 & 102.01 & 101.98 \\
\hline \hline
\end{tabular}

Trade/order size constraints. To explore the effect of boundaries on the optimization results, we include bid/ask sizes for the option trades. By bid/ask size, we mean the amount of each option 
investors are willing to sell/buy at the best available price. We assume that the options expiring in 1 month have a bid/ask size of 500 while the options expiring in 3 months have a bid/ask size of 100, and that the stock has a bid/ask size of 100. The smaller bid/ask size of options expiring later is meant to model the effect of decreased liquidity for longer-dated options. The results of the optimization approaches, with bid/ask size constraints, are presented in Table 5 .

Table 5: Portfolio allocations resulting from optimization algorithms (with bid/ask size constraints).

\begin{tabular}{cccccc}
\multicolumn{7}{c}{ constraints). } \\
\hline \multirow{2}{*}{ Security Type } & $\begin{array}{c}\text { Months to } \\
\text { Expiration }\end{array}$ & $\begin{array}{c}\text { Strike } \\
\text { Price }\end{array}$ & $\begin{array}{c}\text { Scenario } \\
\text { Based }\end{array}$ & $\begin{array}{c}\text { Circle } \\
\text { Extension }\end{array}$ & $\begin{array}{c}\text { Box } \\
\text { Extension }\end{array}$ \\
\hline Call & 1 & $\$ 60$ & 500 & 26 & 37 \\
Call & 3 & $\$ 60$ & 100 & 100 & 100 \\
Put & 1 & $\$ 40$ & 59 & 20 & 21 \\
Put & 3 & $\$ 40$ & 100 & 100 & 100 \\
Stock & & & 19 & 17 & 18 \\
\hline Expected Portfolio Value & & $\$ 102.48$ & $\$ 102.18$ & $\$ 102.26$ \\
\hline \hline
\end{tabular}

The solutions to the optimization end up saturating the bid/ask size constraint in each case. The circle-based extension is able to short more stock and, as a result, purchase more call options.

Sensitivity to bid/ask spreads. So far, we have assumed zero, or negligible, bid/ask spreads. The presence of bid/ask spreads reduces the amount of equity available in the account as a result of transaction costs. Typically shorter dated options have smaller spreads than longer dated options, for the same moneyness. We consider two cases for the bid/ask spreads of the option and equity positions, as outlined in Table 6 .

Table 6: Bid/ask half-spreads as a percentage of market value.

\begin{tabular}{cccc}
\hline \hline Security & $\begin{array}{c}\text { Narrow } \\
\text { Spread Case }\end{array}$ & $\begin{array}{c}\text { Wide } \\
\text { Spread Case }\end{array}$ \\
\hline 1 Month Call $\$ 60$ & $5 \%$ & $25 \%$ \\
3 Month Call $\$ 60$ & $10 \%$ & $40 \%$ \\
1 Month Put $\$ 40$ & $5 \%$ & $25 \%$ \\
3 Month Put $\$ 40$ & $10 \%$ & $40 \%$ \\
Stock & $1 \%$ & $5 \%$ \\
\hline \hline
\end{tabular}


The bid/ask half-spreads are quoted in as a percentage of market value for the option. That is, the bid price is $5 \%$ less than the market value for a one month call option and the ask price is $40 \%$ more than the market value for a three month put option.

Running our optimization algorithm on these securities with these bid/ask spreads produces the results in Table 7.

Table 7: Portfolio allocations resulting from optimization algorithms (with bid/ask spreads).

\begin{tabular}{c|ccccccc}
\hline \hline & \multicolumn{3}{|c}{ Narrow Spread Case } & \multicolumn{3}{c}{ Wide Spread Case } \\
Security & & Scenario & Circle & Box & Scenario & Circle & Box \\
& Based & Extension & Extension & Based & Extension & Extension \\
\hline 1 Month Call $\$ 60$ & 697 & 658 & 615 & 542 & 523 & 607 \\
3 Month Call $\$ 60$ & 1,677 & 1,507 & 1,486 & 1,169 & 1,074 & 1,072 \\
1 Month Put $\$ 40$ & 38 & 51 & 33 & 43 & 42 & -54 \\
3 Month Put $\$ 40$ & -885 & -267 & -207 & -80 & -109 & -148 \\
Stock & 0 & -2 & 0 & 0 & 0 & 0 \\
\hline Expected Portfolio Value & 109.07 & 108.78 & 108.75 & 106.88 & 106.39 & 106.45 \\
\hline \hline
\end{tabular}

Spreads can have a dramatic effect on the optimal portfolios constructed when using the discrete scenario-based approach. Because of the discontinuous nature of the scenario-based approach, the optimal portfolio can be sensitive to the size of the bid/ask spread. The continuous extensions do not exhibit this sensitivity and as such are endowed with more stable results.

Although each portfolio contains large positions in long-term put options, there is also a substantial investment in short-term call options. Since the market prices of options are based upon a positive risk-free rate, it is more likely that call options will end the holding period in the money. The primary investment is long-term put options financed through the shorting of stock. The discrete scenario portfolio has the highest return as compensation for the higher risk.

Different market expectations. To show the effect of different market expectations, we consider an investor who expects a downward trending market. Following a similar setting in the upward trending market, we consider a situation wherein an investor would like to minimize her exposure to an asset using a fixed amount of cash to purchase/short short-term call and put options as well as stock. In this situation, the investor believes that the asset will depreciate relative to the market expectation and as a result the investor would view the stock and call options as expensive and put options as cheap since her view of the stock is generally less favorable than that of the market.

The investor expects the non-dividend paying stock to return $0 \%$ annually, lower than the $3 \%$ annual rate implied by option prices. We saw in the previous example that the investor essentially 
purchases long-term call options and shorts long-term put options in order to leverage her exposure to the underlying asset. The results of the optimization approaches are presented in Table 8 .

Table 8: Portfolio allocations resulting from optimization algorithms.

\begin{tabular}{cccccc}
\hline \hline \multirow{2}{*}{ Security Type } & Months to & Strike & Scenario & Circle & Box \\
Expiration & Price & Based & Extension & Extension \\
\hline Call & 1 & $\$ 60$ & 1,604 & 1,656 & 1,595 \\
Call & 3 & $\$ 60$ & 39 & -91 & -87 \\
Put & 1 & $\$ 40$ & 236 & 254 & 236 \\
Put & 3 & $\$ 40$ & 8,107 & 8,114 & 8,099 \\
Stock & & & -20 & -10 & -10 \\
\hline Expected Portfolio Value & & $\$ 109.57$ & $\$ 108.83$ & $\$ 108.82$ \\
\hline \hline & & & & \\
& & & & \\
& & & & &
\end{tabular}

In this paper, we have introduced a risk-based criterion for portfolio allocation decisions. We studied a risk-based portfolio optimization algorithm inspired by the Chicago Mercantile Exchange's margin requirements. We generalized the discrete margin calculations to continuous alternatives and showed that these alternatives are more computationally efficient and, where analytic pricing formulas exist, analytically tractable. Even though we use a quadratic approximation/expansion of the objective function, the high accuracy of the margin calculation is maintained ${ }^{14}$

We presented several examples which provide evidence that the conventional discrete approach is both less precise and less efficient. The portfolios resulting from the discrete optimization approach are less conservative and require far more computational resources.

Our approach is based on the Black-Scholes setting, but it could be extended to a stochastic volatility setting. The idea of using quadratic approximations to estimate changes in option values is the same. Under a stochastic volatility setting, the option values, first-order Greeks, and second order Greeks need to be computed and used for the optimization. We leave this extension to future research.

By embedding risk-based criteria into portfolio allocation decisions, portfolio managers will be more able to weather changes in portfolio value resulting from market fluctuations over time. The three approaches presented here provide managers with choices to customize their optimization approaches and to objectively approach their portfolio allocation decisions.

\footnotetext{
${ }^{14}$ In some extreme cases, the discrepancies between the quadratic model and original model become large. Under these circumstances, we suggest using higher order approximations for the optimization subproblem. Testing the discrepancy between the quadratic model and the closed-form value is also recommended.
} 


\section{A Option Sensitivities in the Black-Scholes Model}

Although the Black-Scholes formulas for the Greeks can be found in most references, we include the formulas here to ensure notational congruence and for the reader's convenience. Consider a European option expiring $T$ years from now with strike price $K$ on an asset with spot price $S$ and volatility $\sigma$. Assume that the both the underlying assets dividend yield $(q)$ and the risk-free rate $(r)$ are constant and continuously-compounded. The Black-Scholes valuation of a European call option with these characteristics is

$$
C(S, \sigma, K, r, q, T)=S e^{-q T} N\left(d_{+}\right)-K e^{-r T} N\left(d_{-}\right)
$$

and the valuation of a European put option with these characteristics is

$$
P(S, \sigma, K, r, q, T)=K e^{-r T} N\left(-d_{-}\right)-S e^{-q T} N\left(-d_{+}\right)
$$

where

$$
d_{ \pm}=\frac{\ln (S / K)+\left(r-q \pm \sigma^{2} / 2\right) T}{\sigma \sqrt{T}}
$$

and $N$ is the standard normal cumulative distribution function.

Using the chain rule, we compute the following identities that determine the change of a derivative security with respect to fractional changes in the asset price $S \rightarrow S\left(1+R_{S}\right)$ and volatilities $\sigma \rightarrow \sigma\left(1+R_{\sigma}\right)$

$$
\begin{gathered}
\frac{\partial}{\partial R_{S}}=\left(\frac{\partial S}{\partial R_{S}}\right) \frac{\partial}{\partial S}=S \frac{\partial}{\partial S} \text { and } \frac{\partial^{2}}{\partial R_{S}^{2}}=\left(\frac{\partial S}{\partial R_{S}}\right)^{2} \frac{\partial^{2}}{\partial S^{2}}=S^{2} \frac{\partial}{\partial S^{2}} \\
\frac{\partial}{\partial R_{\sigma}}=\left(\frac{\partial \sigma}{\partial R_{\sigma}}\right) \frac{\partial}{\partial \sigma}=\sigma \frac{\partial}{\partial \sigma} \text { and } \frac{\partial^{2}}{\partial R_{\sigma}^{2}}=\left(\frac{\partial \sigma}{\partial R_{\sigma}}\right)^{2} \frac{\partial^{2}}{\partial \sigma^{2}}=\sigma^{2} \frac{\partial^{2}}{\partial \sigma^{2}}
\end{gathered}
$$

The first derivative of the option valuation formulas are given by

$$
\begin{aligned}
\frac{\partial C}{\partial R_{S}} & =S e^{-q T} N\left(d_{+}\right), \quad \frac{\partial P}{\partial R_{S}}=S e^{-q T}\left(N\left(d_{+}\right)-1\right), \\
\frac{\partial C}{\partial R_{\sigma}} & =S e^{-q T} \sigma \sqrt{T} N^{\prime}\left(d_{+}\right)=\frac{\partial P}{\partial R_{\sigma}}
\end{aligned}
$$

and the second derivative of the option valuation formulas are given by

$$
\begin{aligned}
\frac{\partial^{2} C}{\partial R_{S}^{2}} & =\frac{S e^{-q T} N^{\prime}\left(d_{+}\right)}{\sigma \sqrt{T}}=\frac{\partial^{2} P}{\partial R_{S}^{2}}, \\
\frac{\partial^{2} C}{\partial R_{\sigma}^{2}} & =S e^{-q T} \sigma \sqrt{T} d_{+} d_{-} N^{\prime}\left(d_{+}\right)=\frac{\partial^{2} P}{\partial R_{\sigma}^{2}}, \\
\frac{\partial^{2} C}{\partial R_{\sigma} \partial R_{S}} & =-S e^{-q T} d_{-} N^{\prime}\left(d_{+}\right)=\frac{\partial^{2} P}{\partial R_{\sigma} \partial R_{S}} .
\end{aligned}
$$


Because the second partial derivatives of the option formulas are equal between call and put options with the same parameters, higher order derivatives will also coincide. This dramatically reduces the number of analytic option formulas needed when considering higher order changes resulting from fractional changes in the underlying parameters.

\section{References}

S. Alexander, T. F. Coleman, and Y. Li. Minimizing CVaR and VaR for a portfolio of derivatives. Journal of Banking and Finance, 30:583-605, 2006.

N. Branger, B. Breuer, and C. Schlag. Optimal derivative strategies with discrete rebalancings. Journal of Derivatives, 16(2):67-84, 2008.

R. H. Byrd, R. B. Schnabel, and G. A. Schultz. Approximate solution of the trust region problem by minimization over two-dimensional subspaces. Mathematical Programming, 40:247-263, 1988.

E. G. Coffman Jr., D. Matsypura, and V. G. Timkovsky. Portfolio margining: Strategy versus risk. 2010. Working paper, University of Sydney.

C. Dert and B. Oldenkamp. Optimal guaranteed return portfolios and the casino effect. Operations Research, 48(5):768-775, 2000.

J. A. Faias and P. Santa-Clara. Optimal option portfolio strategies. 2011. Working Paper, available at: http://papers.ssrn.com/.

R. H. Filler. Ask the professor - portfolio margining - how will Dodd-Frank impact its utilitzation. Journal of the Law of Investment $\&$ Risk Management, 30:1-6, 2010.

S. Lleo. Risk management: A review. Research Foundation of CFA Institute Literature Review, 4 (1):1-51, 2009.

J. J. Moré and D. C. Sorensen. Computing a trust region step. SIAM Journal on Scientific and Statistical Computing, 4:553-572, 1983.

S. Murray. A margin requirement based return calculation for portfolios of short option positions. Managerial Finance, 39(6):550-568, 2013.

J. Nocedal and S. J. Wright. Numerical Optimization. Springer-Verlag, 1999.

M. J. D. Powell. A new algorithm for unconstrained optimization, pages 31-65. Academic Press, 1970. 
K. M. Rosenzweig. An introduction to portfolio margining. Futures and Derivatives Law Report, 26(11):1-7, 2006.

P. Santa-Clara and A. Saretto. Option strategies: Good deals and margin calls. Journal of Financial Markets, 12:391-417, 2009.

Y. Yamai and T. Yoshiba. Comparative analyses of expected shortfall and value-at-risk: Their estimation error, decomposition, and optimization. Monetrary and Economic Studies, 20(1): 87-122, 2002a.

Y. Yamai and T. Yoshiba. Comparative analyses of expected shortfall and value-at-risk (2): Expected utility maximization and tail risk. Monetrary and Economic Studies, 20(2):95-116, $2002 b$.

L. Zhu, T. F. Coleman, and Y. Li. Min-max robust and CVaR robust mean-variance portfolios. Journal of Risk, 11(3):1-31, 2009.

S. Zymler, B. Rustem, and D. Kuhn. Robust portfolio optimization with derivative insurance guarantees. European Journal of Operational Research, 210(2):410-424, 2011.

S. Zymler, D. Kuhn, and B. Rustem. Worst-case value-at-risk of non-linear portfolios. Management Science, 59(1):172-188, 2013.

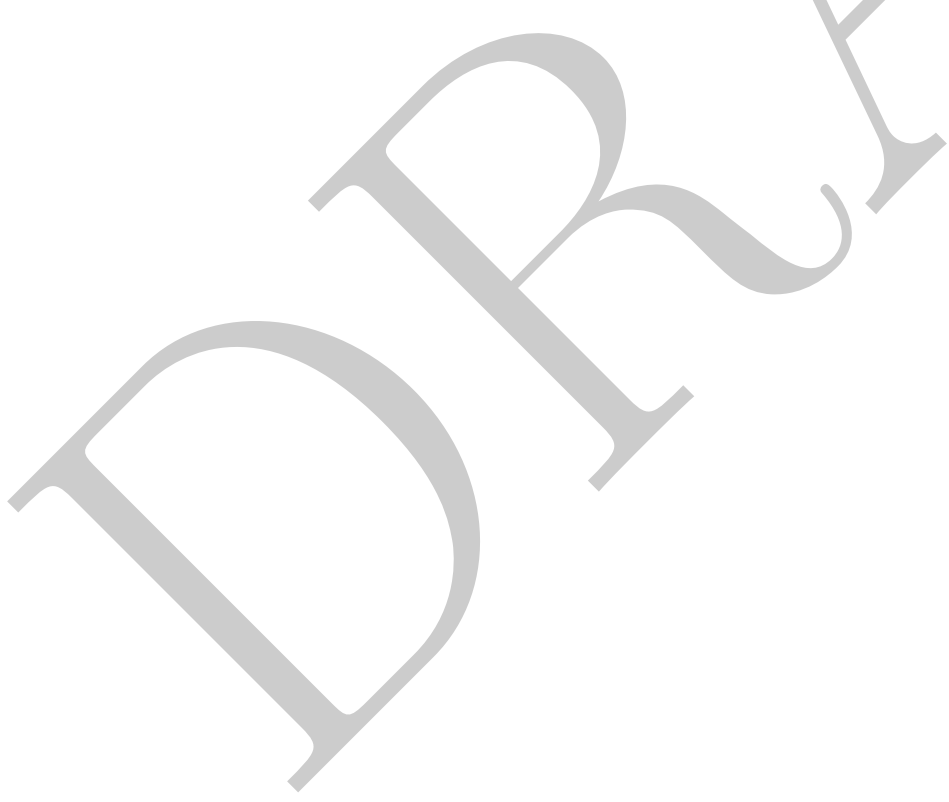

